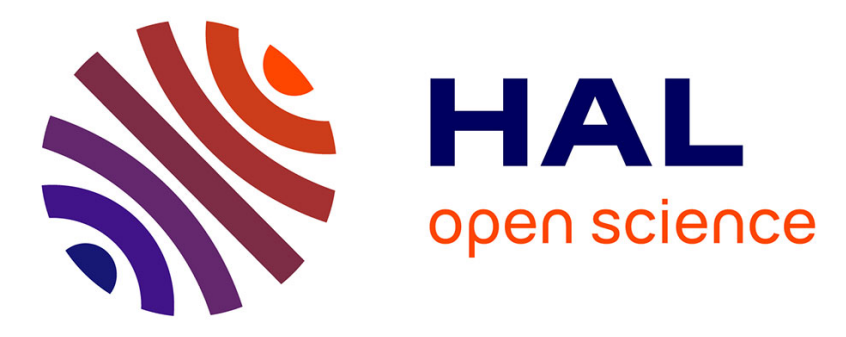

\title{
Simulation-based Optimal Motion Planning for Deformable Object
}

Eiichi Yoshida, Ko Ayusawa, Ixchel Ramirez-Alpizar, Kensuke Harada, Christian Duriez, Abderrahmane Kheddar

\section{To cite this version:}

Eiichi Yoshida, Ko Ayusawa, Ixchel Ramirez-Alpizar, Kensuke Harada, Christian Duriez, et al.. Simulation-based Optimal Motion Planning for Deformable Object. ARSO: Advanced Robotics and its Social Impacts, Jun 2015, Lyon, France. 10.1109/ARSO.2015.7428219 hal-01255803

\section{HAL Id: hal-01255803 \\ https://hal.inria.fr/hal-01255803}

Submitted on 14 Jan 2016

HAL is a multi-disciplinary open access archive for the deposit and dissemination of scientific research documents, whether they are published or not. The documents may come from teaching and research institutions in France or abroad, or from public or private research centers.
L'archive ouverte pluridisciplinaire HAL, est destinée au dépôt et à la diffusion de documents scientifiques de niveau recherche, publiés ou non, émanant des établissements d'enseignement et de recherche français ou étrangers, des laboratoires publics ou privés. 


\title{
Simulation-based Optimal Motion Planning for Deformable Object
}

\author{
Eiichi Yoshida ${ }^{1}$, Ko Ayusawa ${ }^{1}$, Ixchel G. Ramirez-Alpizar ${ }^{2}$, Kensuke Harada ${ }^{2}$, \\ Christian Duriez ${ }^{3}$, and Abderrahmane Kheddar ${ }^{1}$
}

\begin{abstract}
This paper presents a method for planning motions of a flexible objects based on precise simulation using Finite Element Method (FEM). The proposed method is applied to ring-shape objects manipulated by robot arms, which is often seen in various applications. Since large deformation is implied, assembly planning with realistic simulation is important to ensure task efficiency for the robot and also to avoid damage of the object. We first verify that the behavior of a ring-shape object by dual-arm manipulation is well predicted using FEM model of bent beam through a simulation along the trajectory computed by optimization-based motion planning previously reported. Next, a precise FEM model is integrated into optimization to compute a trajectory of robot hands minimizing the deformation energy as well as satisfying such criteria as collision avoidance and smoothness. Since the direct computation leads huge computational cost, we present a realistic formula which transforms the planning problem into the static equilibrium problem of several FEM models located along the trajectory. Simulation results show that the proposed method is promising for such assembly tasks requiring large deformation.
\end{abstract}

\section{INTRODUCTION}

Integration of dexterous robots has been accelerating in part and assembly manipulation in high-mix low-volume production system. Recently, dual-arm robots like Baxter [1] of Rethink Robotics and Nextage [2] of Kawada Industries have been introduced in cellular production systems by sharing the space with human workers. Especially, their dualarm manipulation capacity combined with vision recognition system widens the range of tasks that those robots can execute: picking up parts with complex shape and placing them in other assembled parts through dual-arm motions or regrasping [3]. By delegating simple tasks to robots, humans can concentrate on more complex assembly tasks. The main tasks executed by those robots are currently limited to manipulations that do not involve large deformations of parts. However in factory automation using industrial robots, there still exist some assembly tasks requiring large deformations that need to be automated in manufacturing, such as cable handling, snapping assembly [4] or O-ring assembly [5].

In this paper, we focus on an assembly task to place a ringshape flexible part on a desired position of a cylindrical part by bimanual manipulation (Fig. 1). This type of manipulation often appears in assembly of oil-seals and o-rings in chemical or automobile industries. Previous research has addressed optimization-based motion planning of a ring-shape object

${ }^{1,2}$ E. Yoshida and K. Ayusawa are with CNRS-AIST JRL (Joint Robotics Laboratory), UMI3218/RL, I .Ramirez-Alpizar, K. Harada are with Manipulation Research Group, both Intelligent Systems Research Institute, AIST, Tsukuba, Japan. E-mail: e.yoshida@aist.go. jp

${ }^{3}$ Christian Duriez is with INRIA Shacra Team, 40, avenue Halley, 59650 Villeneuve d'Ascq, France. E-mail: christian.duriezeinria.fr
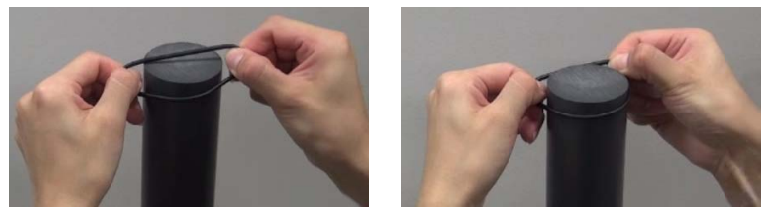

Fig. 1. Assembly of a ring-shape object including deformation

in order to meet criteria of trajectory smoothness, collision avoidance and minimum deformation simultaneously [5]. This work employs a simple model of the ring object by using a set of rigid bodies connected through springs. Likewise, although several methods have been proposed for motion planning of deformable objects [6], [7], [8], [9], [10], they reduce the dimension of the problem or utilize simplified model of deformable objects due to the complexity of the problem. This particular assembly implies necessarily large elastic expansion since we need to consider the space for the hands holding the object. Therefore manipulation motion without excessive deformation is important in order to avoid any damage on the object. Moreover, free deformation is assumed without taking into account the robot hands manipulating the object in previous research. This background motivates us to integrate precise modeling of deformable objects simulating their realistic behavior into assembly motion planning.

In this paper, we propose a method for planning of ringshape objects by using a more precise simulation model based on finite element method (FEM), where manipulating robotic hands are explicitly represented instead of allowing free object motions assumed in most previous work. An optimization-based planning method is first described based on Covariant Hamiltonian Optimization and Motion Planning (CHOMP) method [11], [12] by combining the deformation energy into the cost function. We then introduce a beam FEM model of ring-shape objects to demonstrate the validity of simulation-based motion planning that allows reducing excessive forces applied to the object during manipulation. Finally a precise FEM model is integrated into trajectory optimization problem together with mechanical equilibrium as an additional constraint. An efficient method is presented for solving the complex planning as a static equilibrium problem through decoupling FEM models at each time instance of discretized trajectory. We will demonstrate simulation results to validate the effectiveness the proposed method.

\section{RELATED WORK}

The problem of motion planning for deformable object has started attracting researchers' interest in early 2000s 
when randomized and sampling-based motion planning made a significant progress. Since deformable objects have far more degrees of freedom (DOF) than even complex robotic manipulators, the principal approach is to apply motion planning method to its reduced model. Lamiraux and Kavraki proposed a method for planning thin deformable objects manipulated by two actuators at the edges using samplingbased method in reduced dimensions with pre-computed deformation configurations [6]. Mahoney et al. proposed another approach to extract a reduced basis approximating the deformed configuration space using Principal Component Analysis (PCA) [9].

Rather than converting the problem into a framework whose reduced-dimension is subject to important simplifying assumptions, some methods intrinsically rely on planning strategies that were previously developed for rigid objects such based on the Probabilistic Roadmap Method (PRM) [13] or on the Rapidly-Exploring Random Tree (RRT) [14]. Bayazit et al. [10] take a two-stage PRM-based method that consists in first generating an "approximate path" which may contain collisions with the environment, and second, specifically computing deformations to adapt to such collisions. A more sophisticated method has been proposed later by introducing a volume preservation constraint that is taken into account in order to improve the physical realism of deformations computed based on a mass-spring system representation for efficient computation [7]. Rodriguez et al. proposed a planning method using RRT to deal with kinodynamic planning problem [8] for a deformable object in an environment that is also flexible. Using an extension of the mass-spring representation as a deformation model, a particular care is taken not to lead to unrealistic deformations by considering so-called distance-preserving forces and volume-preserving forces. A hierarchical approach is then employed for deformation computation. Another efficient simplified representation for motion planning of deformable objects using voxels has been proposed [15].

So far we have outlined related work based on samplingbased motion planning combined with models of deformable object simplified to some extent. In general in those previous studies, more attentions have been paid to implement a planning method that exhibit realistically-looking behavior, rather than simulating the true reaction of the deformable objects. Although some of the work like [8] introduced FEMlike method to model the deformation, most of them assume deformable objects that can make free motions generated by forces applied at arbitrary points without considering robotic hands manipulating the objects. In contrast, one of the contributions of this paper is that we explicitly model the robot hands as Dirichlet boundary conditions for FEM computation.

On the other hand, optimization-based motion planning has also been studied more and more intensively. Among them, CHOMP [11], [12] has been proposed as a method that refines the trajectory using covariant gradient techniques with a given cost function to improve the quality of sampled trajectories in a continuous manner. Since we can even start with an initial trajectory in collision, CHOMP is considered to be suitable for highly constrained motion planning problems including narrow passages. For this reason, RamirezAlpizar recently employed CHOMP for motion planning of deformable objects that is also a problem under severe constraints by integrating deformation energy into the cost function [5]. It is, however, based on simplified model of deformable objects. In the following sections, we will present a method with FEM model for deformation to simulate the precise behavior in order to reduce the risk of damages of manipulated objects and to improve the efficiency of assembly.

\section{Simulation-BASED PlanNing}

In this section we integrate FEM simulation to planned trajectory to address such assembly tasks requiring large deformation like ring-shape objects. In our previous research [5], CHOMP was introduced to optimize the trajectory $\xi$ connecting initial and goal configurations $\boldsymbol{q}_{i}$ and $\boldsymbol{q}_{f}\left(\in \Re^{m}\right)$ of the robot hands manipulating the ring-shape object, by formulating the objective function as follows:

$$
\mathcal{U}(\xi)=w_{c} \mathcal{F}_{\text {obs }}(\xi)+w_{s} \mathcal{F}_{\text {smooth }}(\xi)+w_{e} \mathcal{F}_{\text {energy }}(\xi)
$$

where $w_{c}, w_{s}, w_{e}$, are the weights of objective for the obstacle $\mathcal{F}_{\text {obs }}$, smoothness $\mathcal{F}_{\text {smooth }}$ and energy $\mathcal{F}_{\text {energy }}$ respectively. The first two objectives are found in [12] and the last one for energy is based on the deformation energy of the ring-shaped object based on simple model of rigid bodies connected by springs.

In order to confirm the feasibility of the planned trajectories, more realistic simulation model based on FEM has been applied. We adopt SOFA (Software Open Framework Architecture) [16] developed at mainly in INRIA, France. This framework has been developed principally for medical simulation based on real-time FEM computation, but its capacity of simulating various flexible objects allows other dynamic simulations like soft robot control [17] or interacting deformable objects that we are dealing with in this paper.

In this section, we model the ring-shape object as a "bent" beam in FEM model with 40 nodes together with the collision detection with the cylinder. We assume two firm grasping points by robotic hands which are modeled by Dirichlet boundary conditions for FEM as shown in Fig. 2.

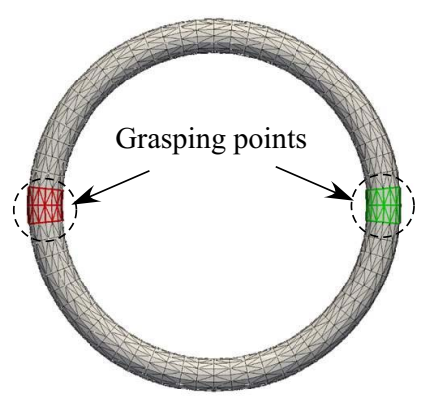

Fig. 2. Grasping points of the ring-shape objects 


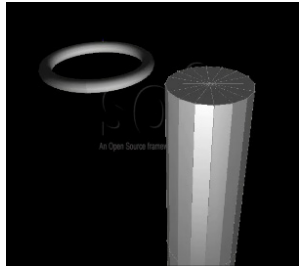

(a) Initial state

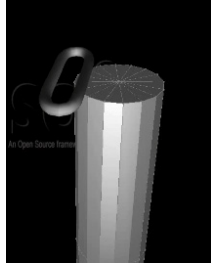

(b)

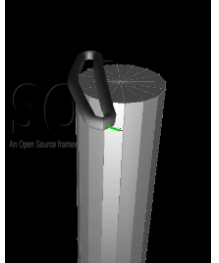

(c)

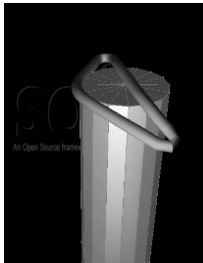

(d)

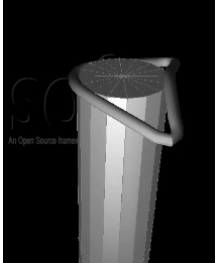

(e)

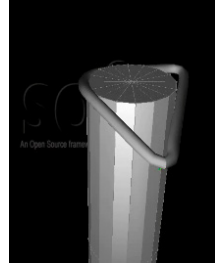

(f)

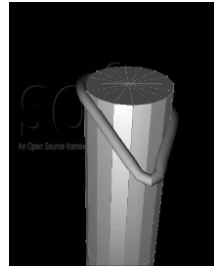

(g) Final state

Fig. 3. Snapshot of ring motion: successful case with optimized trajectory

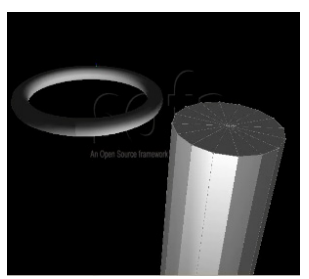

(a) Initial state

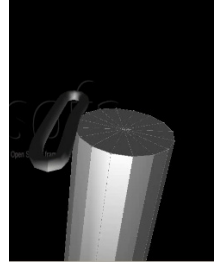

(b)

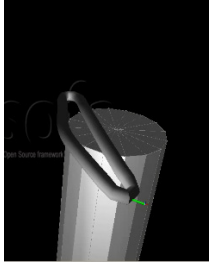

(c)

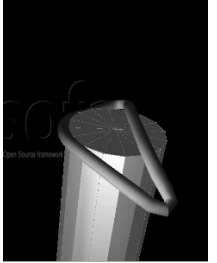

(d)

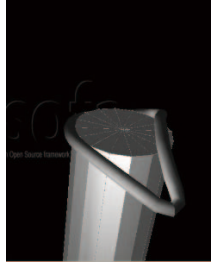

(e)

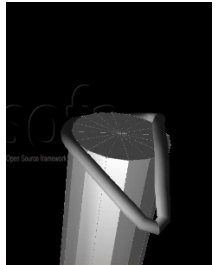

(f)

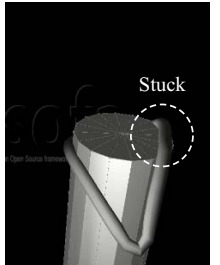

(g) Final state

Fig. 4. Snapshot of ring motion: failure case with non-optimized trajectory

A simple feedback control law is implemented to simulate the manipulations by force-controlled robotic hands whose positions are given as $\boldsymbol{x}_{i}(t)(i=1,2)$ with reference position $\boldsymbol{x}_{i}^{r e f}(t)$ at time $t$.

$$
\boldsymbol{f}_{i}=K\left(\boldsymbol{x}_{i}(t)-\boldsymbol{x}_{i}^{r e f}(t)\right)
$$

Here $K$ is a diagonal matrix specifying the stiffness along each axis of motion. We have implemented a bent beam using FEM model in SOFA and applied the control law.

The assembly task is to place the ring-shape object on the rigid cylindrical part. The inner diameter of the ring is $49 \mathrm{~mm}$ with the cross-section of diameter $3.1 \mathrm{~mm}$ whereas the diameter of cylinder is $50 \mathrm{~mm}$. The Young's modulus of the ring-shape object is $4.1 \mathrm{MPa}$ which corresponds to soft material and we used $3.0 \times 10^{10} \mathrm{~N} / \mathrm{m}$ as the coefficient $K$ in each axis. Figures 3 and 4 show the simulation results with optimized and initial non-optimized trajectory of each grasp point. The latter fails to place the ring-shape object correctly as it is not completely inserted as decribed later. In the figures, we can clearly observe that the ring-shape object is manipulated by the two firm grasping points. The object is pulled first horizontally then vertically to achieve the insertion. Then as can be seen, at the early stage (b) of failure case the object touches the cylinder which is not the case for success case (see the attached video). The vertical downward motion allows the whole ring-shape object to reach around the side surface of the cylinder as seen in Fig. 3(g) in the case of the successful manipulation. On the other hand, the right part of the ring remains stuck on the upper face at the final state in Fig. $4(\mathrm{~g})$ in the unsuccessful case. Thanks to efficient implementation of FEM model in SOFA, here the simulation was almost in real-time with time step of $10 \mathrm{~ms}$ on a processor of Intel(R) Core(TM) i7-4900MQ $\mathrm{CPU}(2.80 \mathrm{GHz})$. Although precise parameter settings like

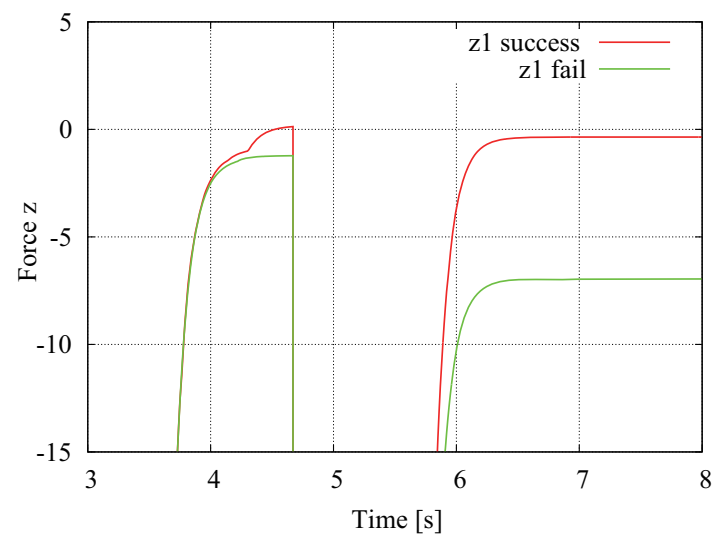

Fig. 5. Vertical force applied to ring-shape object

friction still need to be refined, overall this simulation results demonstrate that precise object modeling is useful to predict the behavior of flexible object for manipulation inducing large deformation.

Figure 5 shows simulated feedback input applied in vertical $(z)$ direction based on control in Eq.(2) at the one of the grasping points. The unit is omitted as the main purpose here is to compare the two simulation results in Figs. 3 and 4. In this graph, we focus on the final state where the difference of two cases is clearly seen. Since the ring-shape object stays expanded even after the manipulation trajectory is finished, the absolute value of force in case of failure case is larger than the successful case. This leads to excessive residual stress to the manipulated object, which should be avoided. The first peak corresponds to the force generated when the left part of the object is inserted and pulled downward at Figs. 3(c) and 4(c). The obtained results show that the validation of planned trajectory based on precise physical simulation using FEM is meaningful for manipulation involving large deformation. 


\section{Optimization-BAsed Planning With FEM SIMULATION}

This section presents the formulation of the optimizationbased motion planning of an FEM object. The computational cost of the optimization problem will be larger because of the large number of variables related to FEM computation. Therefore, we need to approximate the problem with the objective function Eq.(1) to accelerate the computation.

Let us decompose trajectory $\xi$ into discrete time samples as follows:

$$
\boldsymbol{\xi}=\left[\begin{array}{llll}
\boldsymbol{\xi}_{(1)}^{\mathrm{T}} & \boldsymbol{\xi}_{(2)}^{\mathrm{T}} & \cdots & \boldsymbol{\xi}_{(T)}^{\mathrm{T}}
\end{array}\right]
$$

We now consider the following variables at each time instance.

$$
\boldsymbol{\xi}_{(t)}=\left[\begin{array}{ll}
\boldsymbol{d}_{(t)}^{\mathrm{T}} & \boldsymbol{x}_{(t)}{ }^{\mathrm{T}}
\end{array}\right]^{\mathrm{T}}
$$

where, $\boldsymbol{d}$ represents the vector of all positions of each node in an FEM object, and $\boldsymbol{x}$ means the vector of all positions of the grasping points by robotic hands. In this section, we consider the following optimization problem instead of Eq.(1).

$$
\min \mathcal{U}(\boldsymbol{\xi}) \quad \text { subject to } \boldsymbol{y}(\boldsymbol{\xi})=\mathbf{0}
$$

where, $\boldsymbol{y}$ represents the equality constraints related to FEM simulation.

The stationary condition of problem (5) is as follows:

$$
\left(\frac{\partial \mathcal{U}}{\partial \boldsymbol{\xi}}\right)^{\mathrm{T}}+\left(\frac{\partial \boldsymbol{y}}{\partial \boldsymbol{\xi}}\right)^{\mathrm{T}} \boldsymbol{\lambda}=\mathbf{0}
$$

where $\lambda$ is Lagrange multiplier. In order to optimize trajectory $\xi$, we solve Eq.(6), for example, by Newton-Raphson method.

We now detail the each component in problem (5). In this paper, we consider the following boundary conditions as constraint $\boldsymbol{y}(\boldsymbol{\xi})$ :

$$
\begin{gathered}
\boldsymbol{y}_{S} \triangleq \boldsymbol{x}_{(1)}-\boldsymbol{x}_{S}=\mathbf{0} \\
\boldsymbol{y}_{E} \triangleq \boldsymbol{x}_{(T)}-\boldsymbol{x}_{E}=\mathbf{0} \\
\boldsymbol{y}_{f} \triangleq \boldsymbol{d}_{f(t)}-\boldsymbol{r}\left(\boldsymbol{x}_{(t)}\right)=\mathbf{0}
\end{gathered}
$$

where, $\boldsymbol{x}_{S}$ and $\boldsymbol{x}_{E}$ represents the initial and final positions of the grasping points respectively, $\boldsymbol{d}_{f}$ is the vector of all positions of constrained nodes, and $\boldsymbol{r}(\boldsymbol{x})$ means the forward kinematics mapping of $\boldsymbol{d}_{f}$ with respect to $\boldsymbol{x}$. The following relationship holds between $\boldsymbol{d}_{f}$ and $\boldsymbol{d}$.

$$
\boldsymbol{d}_{f}=\boldsymbol{S d}
$$

where, $\boldsymbol{S}$ is the selection matrix which extracts $\boldsymbol{d}_{f}$ from $\boldsymbol{d}$,

We now implement smoothness objective $\mathcal{F}_{\text {smooth }}(\xi)$ as follows:

$$
\mathcal{F}_{\text {smooth }}(\xi)=\sum_{t=2}^{T} l_{(t, t-1)}+\sum_{t=2}^{T-1} e_{(t)}
$$

where,

$$
\begin{aligned}
& l_{(t, t-1)} \triangleq \omega_{l}\left\|\boldsymbol{x}_{(t)}-\boldsymbol{x}_{(t-1)}\right\| \\
& e_{(t)} \triangleq \omega_{e}\left\|\boldsymbol{x}_{(t)}-\boldsymbol{x}_{(t)}{ }^{i n i t}\right\|^{2}
\end{aligned}
$$

The first term in Eq.(11) represents the length of the whole trajectory. The squared distance from the initial trajectory $\boldsymbol{x}_{(t)}{ }^{\text {init }}$ is added in the second term for the computational stability. $\omega_{l}$ and $\omega_{e}$ are the weighting factors.

In obstacle objective $\mathcal{F}_{\text {smooth }}(\xi)$, we also consider the collision condition between the FEM object (i.e. the O-ring) and the other environment (i.e. the cylinder). In this paper, we approximate the collision conditions as the penalty functions against penetration.

$$
\mathcal{F}_{o b s}(\xi)=\sum_{t} E_{p(t)} \triangleq \sum_{t} \omega_{p} E_{p}\left(\boldsymbol{d}_{(t)}\right)
$$

where, $E_{p}(\boldsymbol{d})$ represents the collision penalty function, and $\omega_{p}$ is its weighting factor. Let the energy function be implemented by

$$
\mathcal{F}_{\text {energy }}(\xi) \approx \sum_{t} E_{e(t)} \triangleq \sum_{t} \omega_{e} E_{e}\left(\boldsymbol{d}_{(t)}\right)
$$

where, $E_{e}(\boldsymbol{d})$ is the elastic energy of the FEM object, and $\omega_{e}$ is its weighting factor.

In the original implementation of CHOMP, $\mathcal{F}_{o b s}(\xi)$ is the integrated penalty functions along trajectory $\xi$. In this paper, we approximate both $\mathcal{F}_{\text {obs }}(\xi)$ and $\mathcal{F}_{\text {energy }}(\xi)$ as the summation of the individual corresponding functions. With the approximation, stationary condition (6) can be written as follows:

$$
\begin{gathered}
\boldsymbol{y}_{d(t)} \triangleq \boldsymbol{g}\left(\boldsymbol{d}_{(t)}\right)+\boldsymbol{S}^{\mathrm{T}} \boldsymbol{f}_{(t)}=\mathbf{0} \\
\boldsymbol{y}_{x(t)} \triangleq \boldsymbol{h}_{(t)}+\boldsymbol{J}_{(t)}^{\mathrm{T}} \boldsymbol{f}_{(t)}+\delta_{t, 1} \boldsymbol{\lambda}_{S}+\delta_{t, T} \boldsymbol{\lambda}_{E}=\mathbf{0}
\end{gathered}
$$

where, $f$ means the vector of the constraint forces acting on the constrained nodes of the FEM objects, $\boldsymbol{\lambda}_{S}$ and $\boldsymbol{\lambda}_{E}$ are Lagrange multipliers related to the initial condition, $\boldsymbol{J} \triangleq \frac{\partial \boldsymbol{r}}{\partial \boldsymbol{x}}$, and $\delta_{i, j}$ is Kronecker's delta symbol. $\boldsymbol{g}_{(t)}$ and $\boldsymbol{h}_{(t)}$ are the forces defined as follows:

$$
\begin{gathered}
\boldsymbol{g}(\boldsymbol{d}) \triangleq\left(\frac{\partial E_{e}}{\partial \boldsymbol{d}}\right)^{\mathrm{T}}+\left(\frac{\partial E_{p}}{\partial \boldsymbol{d}}\right)^{\mathrm{T}} \\
\boldsymbol{h}_{(t)} \triangleq\left(\frac{\partial l_{(t-1, t)}}{\partial \boldsymbol{d}_{(t)}}\right)^{\mathrm{T}}+\left(\frac{\partial l_{(t, t+1)}}{\partial \boldsymbol{d}_{(t)}}\right)^{\mathrm{T}}+\left(\frac{\partial e_{(t)}}{\partial \boldsymbol{d}_{(t)}}\right)^{\mathrm{T}}
\end{gathered}
$$

It should be noted that $\boldsymbol{g}$ represents the standard static equilibrium formula of the FEM object under the given boundary conditions. Therefore, Eq.(16) is independent of other time instances; on the other hand, Eq.(17) is dependent. When solving Eq.(16) and Eq.(17) by Newton-Rapthon method, we have to compute the Hessian matrix of the augmented Lagrangian function of problem (5).

Let us redefine the trajectory including Lagrange multipliers as follows:

$$
\widehat{\boldsymbol{\xi}}=\left[\begin{array}{llllll}
\boldsymbol{\lambda}_{S}{ }^{\mathrm{T}} & \widehat{\boldsymbol{\xi}}_{(1)}^{\mathrm{T}} & \widehat{\boldsymbol{\xi}}_{(2)}^{\mathrm{T}} & \cdots & \widehat{\boldsymbol{\xi}}_{(T)}^{\mathrm{T}} & \boldsymbol{\lambda}_{E}^{\mathrm{T}}
\end{array}\right]
$$

where,

$$
\widehat{\boldsymbol{\xi}}_{(t)}=\left[\begin{array}{lll}
\boldsymbol{d}_{(t)}^{\mathrm{T}} & \boldsymbol{x}_{(t)}^{\mathrm{T}} & \boldsymbol{f}_{(t)}^{\mathrm{T}}
\end{array}\right]^{\mathrm{T}}
$$

We also concatenate all the stationary conditions and the constraints as follows:

$$
\boldsymbol{y}=\left[\begin{array}{llllll}
\boldsymbol{y}_{S}{ }^{\mathrm{T}} & \boldsymbol{y}_{(1)}^{\mathrm{T}} & \boldsymbol{y}_{(2)}^{\mathrm{T}} & \cdots & \boldsymbol{y}_{(T)}^{\mathrm{T}} & \boldsymbol{y}_{E}^{\mathrm{T}}
\end{array}\right]
$$




$$
\boldsymbol{y}_{(t)}=\left[\begin{array}{lll}
\boldsymbol{y}_{d(t)}^{\mathrm{T}} & \boldsymbol{y}_{x(t)}^{\mathrm{T}} & \boldsymbol{y}_{f(t)}^{\mathrm{T}}
\end{array}\right]
$$

The Hessian matrix can be written as follows:

$$
\boldsymbol{H} \triangleq\left[\begin{array}{ccccccc}
\boldsymbol{O} & \boldsymbol{E} & \boldsymbol{O} & \cdots & \boldsymbol{O} & \boldsymbol{O} & \boldsymbol{O} \\
\boldsymbol{E} & \boldsymbol{H}_{1} & \boldsymbol{G}_{1,2} & \cdots & \boldsymbol{O} & \boldsymbol{O} & \boldsymbol{O} \\
\boldsymbol{O} & \boldsymbol{G}_{2,1} & \boldsymbol{H}_{2} & \cdots & \boldsymbol{O} & \boldsymbol{O} & \boldsymbol{O} \\
\vdots & \vdots & \vdots & \ddots & \vdots & \vdots & \boldsymbol{O} \\
\boldsymbol{O} & \boldsymbol{O} & \boldsymbol{O} & \cdots & \boldsymbol{H}_{T-1} & \boldsymbol{G}_{T-1, T} & \boldsymbol{O} \\
\boldsymbol{O} & \boldsymbol{O} & \boldsymbol{O} & \cdots & \boldsymbol{G}_{T, T-1} & \boldsymbol{H}_{T} & \boldsymbol{E} \\
\boldsymbol{O} & \boldsymbol{O} & \boldsymbol{O} & \cdots & \boldsymbol{O} & \boldsymbol{E} & \boldsymbol{O}
\end{array}\right]
$$

where, $\boldsymbol{E}$ represents the identify matrix. $\boldsymbol{H}_{t}$ and $\boldsymbol{G}_{t, s}$ are defined by

$$
\begin{gathered}
\boldsymbol{H}_{t}=\left[\begin{array}{ccc}
\frac{\partial \boldsymbol{g}_{(t)}}{\partial \boldsymbol{d}_{(t)}} & \boldsymbol{O} & \boldsymbol{S}^{\mathrm{T}} \\
\boldsymbol{O} & \frac{\partial \boldsymbol{h}_{(t)}}{\partial \boldsymbol{x}_{(t)}} & \boldsymbol{J}_{(t)}^{\mathrm{T}} \\
\boldsymbol{S} & \boldsymbol{J}_{(t)} & \boldsymbol{O}
\end{array}\right] \\
\boldsymbol{G}_{t, s}=\left[\begin{array}{ccc}
\boldsymbol{O} & \boldsymbol{O} & \boldsymbol{O} \\
\boldsymbol{O} & \frac{\partial \boldsymbol{h}_{(t)}}{\partial \boldsymbol{d}_{(s)}} & \boldsymbol{O} \\
\boldsymbol{O} & \boldsymbol{O} & \boldsymbol{O}
\end{array}\right]
\end{gathered}
$$

As can be seen from Eq. (25), all the columns and rows related to the FEM variables are decoupled each other; the columns and rows related to the rigid-body variables at each time instance are coupled with those of the previous and the following time instance. Since the number of variables related to the rigid-body part is quite smaller than that of the FEM variables, the computational cost at each iteration of the Newton-Rapthon method is almost the same as when we solve independent FEM equations at $T$ time instances. In addition, all the components in $\boldsymbol{H}$ and $\boldsymbol{y}$ can be obtained from the standard computation of FEM and rigid-body equations respectively. In other word, the motion-planning problem is transformed into the huge static equilibrium problem of $T$ objects whose grasping points are connected via virtual springs along with the trajectory.

We tested the proposed optimization formula by using the ring-shape object and the grasping points shown in Fig. 2, and the same environmental setting as that in section III. In this section, the ring-shape object was expressed with tetrahedral 3D meshes whose number of nodes is 1088 and number of elements is 3072 , in order to test the proposed scheme for general FEM models. The O-ring was modeled using a hyper-elastic material (Mooney-Revlin). We also consider the quasi-incompressibility condition of the ring object in the simulation; the pressures inside the elements of the object were also taken into account by adding them in vector $\boldsymbol{d}$ as new variables. We utilized FEM solver V-biomech [18] in order to compute the terms of nonlinear FEM model used in the optimization. The trajectory was discretized by $T=10$ samples, and the initial trajectory before optimization was given as shown in Fig. 6. Since the initial trajectory was
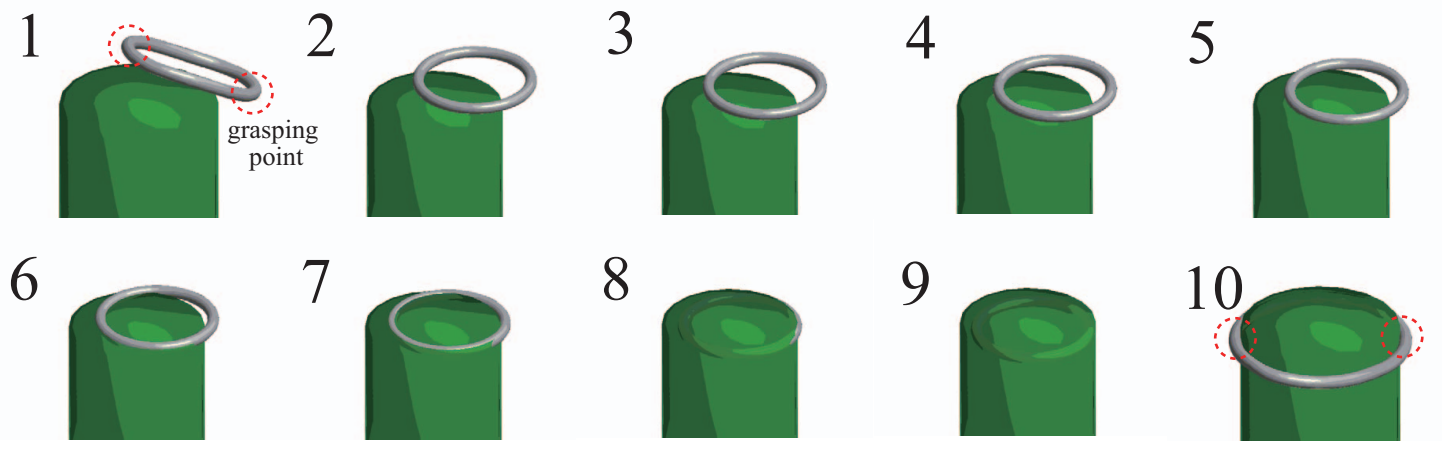

Fig. 6. Snapshot of initial trajectory of ring motion before optimization
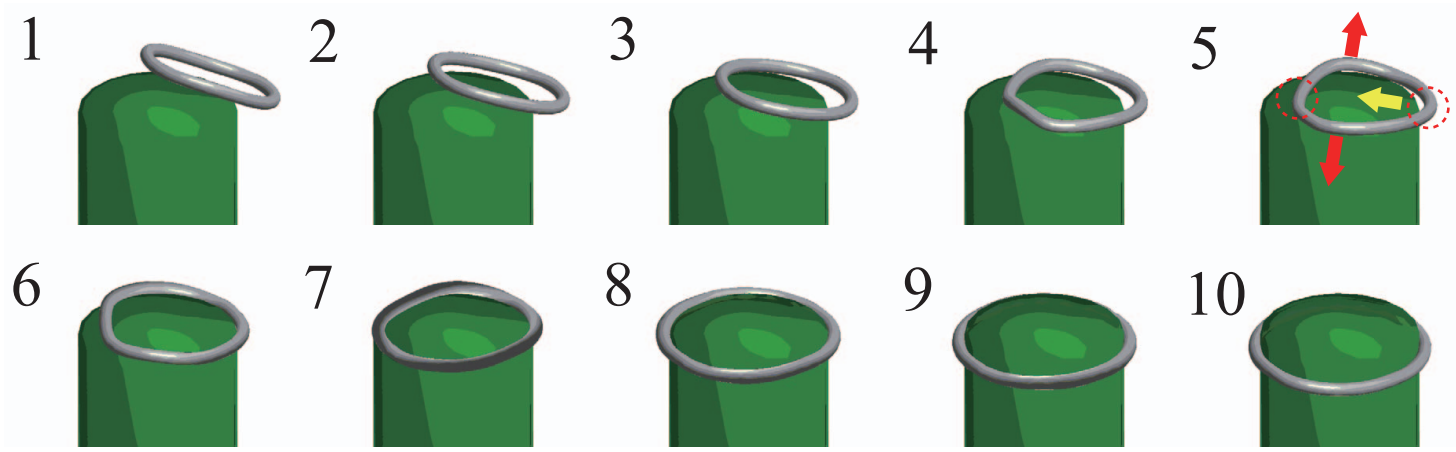

7

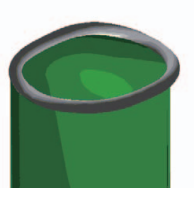

8

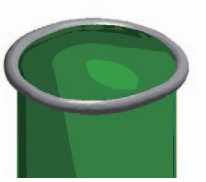

9

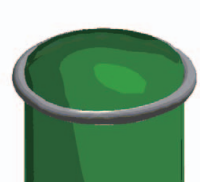

10

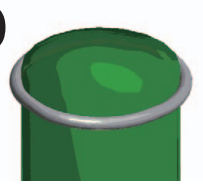

Fig. 7. Snapshot of obtained trajectory of ring motion after optimization 
just interpolated linearly without considering deformation, the ring penetrated inside the cylinder during the motion. The method was implemented on the workstation with the same CPU used in section III. As we mentioned, our framework is equivalent to solving huge static equilibrium problem of $T$ FEM objects. Since $i$-th FEM object is only coupled with $(i-1)$-th and $(i+1)$-th objects as shown in Eq.(25), we can utilize this sparseness when we solve Eq.(24). Therefore, the computational complexity to solve Eq.(24) is almost linear with respect to the number of time samples. Finally, the total computation time of the whole optimization was around 30 minutes.

Fig. 7 shows the obtained trajectory after the optimization, which resulted in another strategy to achieve the insertion from section III. The left grasping point in the figure remained fixed on the top of the cylinder during first time samples, and the right grasping point went down and close to the side of cylinder, which leads to the increase of the internal length of the ring along the direction vertical to this paper (Fig. 7, step 5). After hooking the half part of the ring on the cylinder, the left grasping point passed over the cylinder without collision, and went down to the final position to achieve the insertion.

Although the pilot optimization framework can obtain the local optimal solution according to the objective functions, it does not guarantee the global optimal solution. For this purpose we need another framework to explore the candidate of the initial guess of the trajectory, for example, by a simpler simulation as shown in section III. The current optimization also approximates the integration of the objective function along with the trajectory by the simple summation. Those issues will be investigated in our future works.

\section{Conclusion and Future Work}

This paper presented a simulation-based optimal motion planning method for assembly of a flexible ring-shape object including large deformation. After introducing an optimization-based motion planning called CHOMP that can integrate objective functions of minimum energy manipulation, realistic simulation model based on FEM, together with the collision detection, was applied to verify the planned trajectory. The ring-shape object was represented by a bent beam firmly grasped by robotic hands of a dual-arm manipulator. By comparing the force applied to the object for non-optimized and optimized trajectories, we could observe that the latter was successful without excessive manipulation force. This simulation showed the significance of realistic simulations instead of reduced models in previous work. Next we presented optimization-based motion planning integrating the more accurate FEM model containing more than 1000 nodes by using objective functions of smoothness, collision avoidance and minimum deformation energy under static equilibrium conditions. An efficient computation scheme was derived to solve the planning problem as a static equilibrium problem of decoupled FEM objects at each time instance in a discretized model of the trajectory optimization. Simulation results demonstrated that the proposed method can provide plausible behaviors of deformable ring-shape object to plan the trajectory for the robotic hands.

Future work includes the refinement of the proposed optimization-based method. As we have discretized the trajectory and used the sum of objective function instead of integration, global trajectory optimization scheme with even more efficient FEM computation will be one of the forthcoming stages of this research. The verification of the planned trajectory with experiments with a real robot is also another future topic. We intend to confirm the predicted behavior from the simulation and to improve the model based on the experimental results.

\section{ACKNOWLEDGMENTS}

We would like to express our sincere gratitude to $\mathrm{Mr}$. TuHoa Pham, currently a Ph.D candidate at CNRS-AIST JRL, for his contribution to discussion on motion planning and mechanical computing.

\section{REFERENCES}

[1] http://www.rethinkrobotics.com/baxter/, 2015.

[2] http://nextage.kawada.jp/, 2015.

[3] http://www.glory.co.jp/company/news/2012/1011.html, (in Japanease).

[4] J. Rojas, K. Harada, H. Onda, N. Yamanobe, E. Yoshida, K. Nagata, and Y. Kawai, "Towards snap sensing," Int. J. Mechatronics and Automation, vol. 3, no. 2, pp. 69-93, 2013.

[5] I. G. Ramirez-Alpizar, K. Harada, and E. Yoshida, "Motion planning for dual-arm assembly of ring-shaped elastic objects," in Proc. 2014 IEEE-RAS Int. Conf. on Humanoid Robots, 2014, pp. 594-600.

[6] F. Lamiraux and L. E. Kavraki, "Planning paths for elastic objects under manipulation constraints," Int. J. Robotics Research, vol. 20, no. 3, pp. 188-208, 2001.

[7] R. Gayle, M. C. Lin, and D. Manocha, "Constraint-based motion planning of deformable robots," in Proc. 2005 IEEE Int. Conf. on Robotics and Automation, 2005, pp. 1046-1053.

[8] S. Rodriguez, J.-M. Lien, and N. M. Amato, "Planning motion in completely deformable environments," in Proc. 2006 IEEE Int. Conf. on Robotics and Automation, 2006, pp. 2466-2471.

[9] A. Mahoney, J. Bross, and D. Johnson, "Deformable robot motion planning in a reduced-dimension configuration space," in Proc. 2007 IEEE Int. Conf. on Robotics and Automation, 2010, pp. 5133-5188.

[10] B. Bayazit, J.-M. Lien, and N. M. Amato, "Probabilistic roadmap motion planning for deformable objects," in Proc. 2002 IEEE Int. Conf. on Robotics and Automation, 2002, pp. 2126-2133.

[11] N. Ratliff, M. Zucker, J. A. Bagnell, and S. Srinivasa, "CHOMP: Gradient optimization techniques for efficient motion planning," in Proc. 2009 IEEE Int. Conf. on Roboics and Automation, 2009, pp. 489-494.

[12] M. Zucker, N. Ratliff, A. D. Dragan, M. Pivtoraiko, M. Klingensmith, C. M. Dellin, J. A. Bagnell, and S. S. Srinivasa, "CHOMP: Covariant hamiltonian optimization for motion planning," Int. J. Robotics Research, vol. 32, no. 9-10, pp. 1164-1193, 2013.

[13] L. Kavraki, P. Svestka, J.-C. Latombe, and M. Overmars, "Probabilistic roadmaps for path planning in high-dimensional configuration spaces," IEEE Trans. on Robotics and Automation, vol. 12, no. 4, pp. 566-580, 1996.

[14] S. LaValle and J. Kuffner, "Rapidly-exploring random trees: Progress and prospects," in Algorithmic and Computational Robotics: New Directions, K. M. Lynch and D. Rus, Eds. A K Peters, 2001, pp. 293-308.

[15] C. Phillips-Grafflin and D. Berenson, "Representation of deformable objects for motion planning with no physical simulation," in Proc. 2014 IEEE Int. Conf. on Robotics and Automation, 2014, pp. 98-105.

[16] http://www.sofa-framework.org/.

[17] C. Duriez, "Control of elastic soft robots based on real-time finite element method," in Proc. 2013 IEEE Int. Conf. on Robotics and Automation, 2013, pp. 3982-3987.

[18] J.L.Alves, N.Yamamura, T.Oda, and C.Teodosiu, "Numerical simulation of musculo-skeletal systems by V-biomech," 2010. 